\title{
Miniaturized Cold Atmospheric Plasma for the Conservation of Plastics in Modern and Contemporary Art
}

\author{
Anna Comiotto*
}

\begin{abstract}
In order to overcome adhesion problems during conservation and restoration treatments on non-polar plastics in modern and contemporary art, a miniaturized cold plasma source was developed. This source can be operated without vacuum at atmospheric pressure, has a sphere of action in the millimetre range and is tailored to the application on heat-sensitive plastics. In this article, the setup of the developed plasma equipment is described and examination results concerning the effectiveness of this pre-treatment for enhancing the wettability, bondability and coatability of poly(ethylene), poly(propylene) and poly(styrene) are presented. Furthermore, experiments for reducing the thermal load during the pre-treatment of heat-sensitive polymers are specified. The effectiveness of this pre-treatment has been proved by bonding low-density poly(ethylene) [PE-LD], high-density poly(ethylene) [PE-HD] and poly(propylene) [PP] with the acrylic resin Acryloid B-72, whereas the extent of adhesion improvement was quantified by performing tensile shear tests. Changes in coatability were examined in pull-off tests, carried out on gouache painted PE-HD and poly(styrene) [PS]. For a better understanding of the induced chemical changes, treated and untreated PP surfaces were examined by water contact angle measurements and chemically characterized by surface-sensitive infrared Spectroscopy (FTIR-ATR). Beside an observed enhancement in water wettability and surface polarity, after the pre-treatment the strength of all tested adhesive bonds was significantly enhanced. The applied gouache paint coatings also gained a considerable mechanical resilience; therefore the application of durable, still reversible retouchings on hydrophobic, low surface energy polymers becomes possible. Further experiments will be necessary in order to exclude undesirable side effects on the long-term aging properties of the pre-treated polymers, especially with respect to their oxidative stability.
\end{abstract}

Keywords: Adhesion improvement · Modern and Contemporary Art · Plasma

\section{Introduction}

Synthetic polymers have become an essential part of modern life and artists have included these new materials in their working process. Unfortunately, some plastics in works of modern and contemporary art, in particular non-polar polymers like poly(ethylene) [PE] and poly(propylene) [PP], are known to have insufficient adhesion properties.

${ }^{\star}$ Correspondence: A. Comiotto,

Conservator/Restorer (FH)

Berne University of Applied Sciences, University of the Arts

Department of Conservation and Restoration

Modern Materials and Media

Fellerstrasse 11

$\mathrm{CH}-3027$ Bern

Tel. +41318483873

E-mail: anna.comiotto@hkb.bfh.ch
Due to a lack of surface polarity, they are difficult to wet, their adhesive bonds have limited tensile strength and applied coatings do not possess enough mechanical resilience. As a consequence, durable conservation and restoration treatments (e.g. paint layer consolidation, bonding or retouching) are often not applicable. To improve the adhesion properties of non-polar plastics, a pre-treatment is necessary to provide the polymer surface with an artificial polarity. It is well known that plasma, a high-energy (partially) ionized gas, can enhance surface polarity by creating polar functional groups at polymer surfaces. Various authors have observed this by using surface-sensitive analytic methods. ${ }^{[1-5]}$ As a result of chemical surface modification plasma treatments can improve wettability, coatability and bondability of non-polar plastics. ${ }^{[6-8]}$ The aim of the ongoing research project presented here is to develop a plasma source tailored to art conservation-restoration applications, designed to meet the following demands:

i) The source is operable without vacuum at atmospheric pressure. ii) The plasma is locally applicable in the millimetre range and causes a minimal thermal load of the pre-treated surfaces.

iii) The pre-treatment effects a reproducible and effective improvement of adhesion qualities.

iv) A minimal intervention into the artwork's material integrity should be assured, especially concerning changes in long-term aging properties.

In this paper, the setup of the developed plasma equipment is described and examination results related to changes in wettability, surface polarity, bondability and coatability are presented. Furthermore, experiments to reduce the thermal load during the pre-treatment of heat-sensitive polymers are specified.

\section{Experimental}

\subsection{Plasma Operation}

The plasma source (Fig. 1) works under atmospheric pressure and has a sphere of 
action in the millimetre range. The instrumental setup for operating the plasma is schematically depicted in Fig. 2. A variable AC transformer, coupled to a matched, low cost high-voltage circuit serves as power supply (1). The main part of the pen-shaped plasma source (3) is an alumina capillary (4). In this capillary, the plasma is generated from dielectric barrier discharges. A cross-section through the capillary (5) shows the concentric electrode configuration used $(\mathrm{a}-\mathrm{d})$. The alumina capillary $(\mathrm{c})$ serves as insulating layer (dielectric barrier) between two electrodes (b/d): The primary high-voltage electrode (b) is a thin metal wire, mounted coaxially in the centre of the capillary. A metallization layer serves as a secondary grounded electrode (d). If high voltage is applied to the metal wire, electrical discharges are generated in the gap between the electrodes (a). Provided that the source is operated in the frequency range of at about $6.5 \mathrm{kHz}$ and voltage inputs higher as about 800 volts as well as a suitable process gas are used, a plasma jet effuses out of the capillary (displayed in Fig. 1 under magnification). The process gas (e.g. helium) is fed into the plasma source by letting it pass through a supply pipe (2). The gas throughput can be regulated with gas flow control units. The length of the plasma jet is adjustable within a range of about $1-20 \mathrm{~mm}$ by either changing the high-voltage input or the gas flow rate.

For sample pre-treatment a simple computer-controlled, step motor driven $\mathrm{x}$-y unit (from an $x-y$ plotter) was used. The plotter, fitted with a sample platform, moved the sample along the $\mathrm{x}$ - or $\mathrm{y}$ axis on a pretreatment area of $10 \times 10 \mathrm{~mm}$ at a defined speed. If not otherwise specified, after pretreating the sample for $1 \mathrm{sec}$, a movement of $1 \mathrm{~mm}$ took place in the $\mathrm{x}-$ or $\mathrm{y}$ axis. The plasma source was mounted rectangularly to the moving sample and was kept at an operating distance of $3 \mathrm{~mm}$. Except for temperature measurements, this setup and procedure was applied to all described experiments. For the examination of changes in wettability, polarity, bondability and coatability sample pre-treatment was performed with a voltage input of about $1600 \mathrm{~V}$. If not otherwise specified, helium was used as process gas (gas flow rate: $1000 \mathrm{ml} / \mathrm{min}$ ), under admixture of $0.5 \%$ oxygen.

\subsection{Examination of Changes in Wettability and Surface Polarity}

Changes in wettability and surface polarity after pre-treatment were examined on PP films (Homopolymer, $270 \mu \mathrm{m}$, Goodfellow Research Materials $\mathrm{GmbH}$, Bad Nauheim).

Water wettability was quantified with a contact angle goniometer using the sessile drop method directly after the pre-treatment. During each measurement a drop of

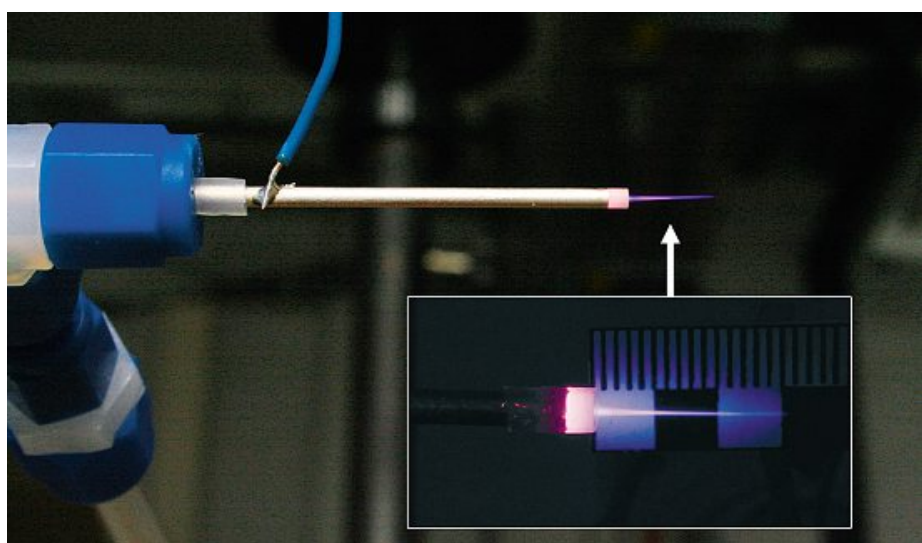

Fig. 1. Photograph of the cold plasma source by displaying the plasma jet under magnification (one mark on the scale corresponds to $1 \mathrm{~mm}$ )

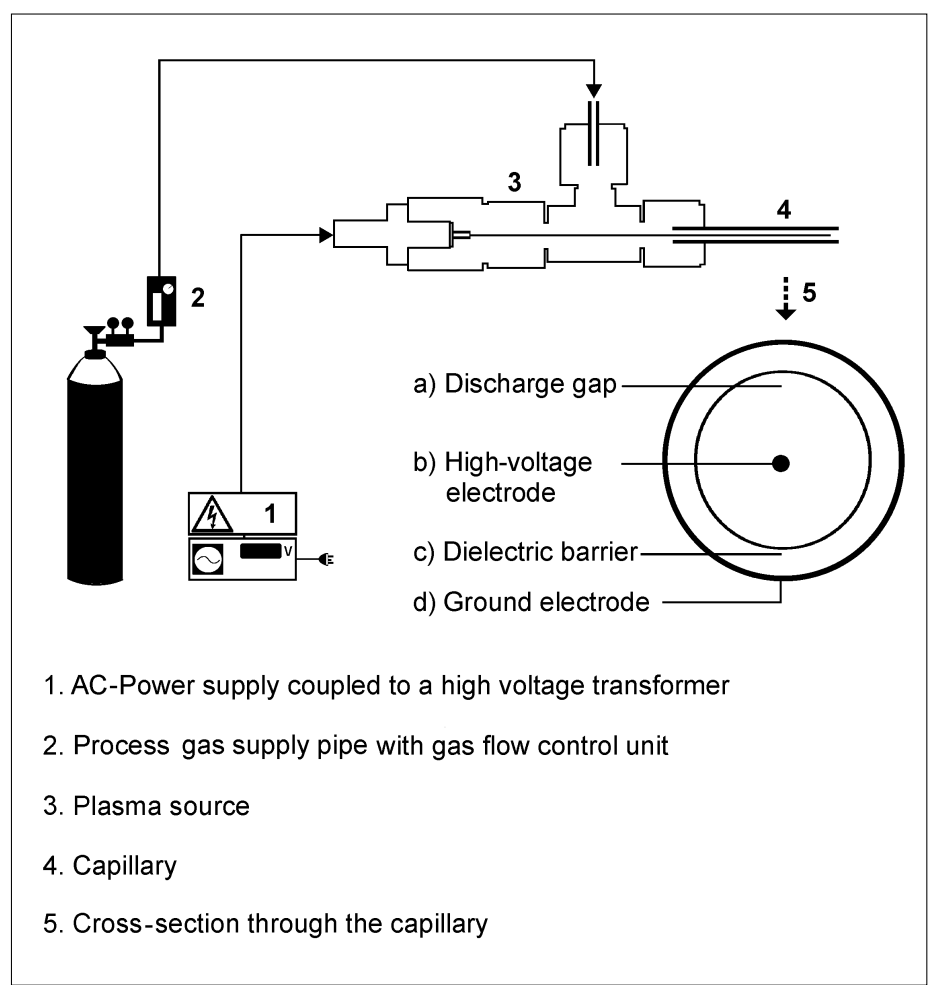

Fig. 2. Experimental setup of the plasma equipment

deionised water with a volume of $5 \mu \mathrm{l}$ was placed on the film surface and a second drop of $5 \mu$ was centered on the first. The advancing contact angles were determined within $60 \mathrm{sec}$ after the first contact of the liquid with the surface.

To gain some understanding on the induced chemical changes, especially with regard to alterations in surface polarity, surface-sensitive infrared spectrometric measurements (FTIR-ATR) were carried out. Infrared spectra of the PP surface were obtained $6 \mathrm{~h}$ after pre-treatment using a Fourier transform infrared spectrometer Vertex 70 from Bruker $\left(2 \mathrm{~cm}^{-1}\right.$ resolution, 64 scans). For semi-quantitative evaluation, peak intensities were normalized by adjusting the intensity of the asymmetric and symmetric $\mathrm{CH}_{2}$-stretch vibrations $\left(2922 \mathrm{~cm}^{-1} / 2840 \mathrm{~cm}^{-1}\right)$ and the asymmetric and symmetric $\mathrm{CH}_{3}$-stretch vibrations (2955 $\mathrm{cm}^{-1} / 2878 \mathrm{~cm}^{-1}$ ).

\subsection{Examination of Changes in Bondability}

To quantify the extent of adhesion improvement, tensile shear tests on glued PE-LD, PE-HD and PP sample joints were performed. As adhesive the acrylic resin Paraloid B-72 was used. This ethylmethacrylate-methylacrylate copolymer is frequently used in art conservation treatments, because of its estimated good long-term aging properties. For sample preparation, commercially extruded PE-LD, PE-HD and PP homopolymers were cut into small plates of $80 \times 10 \times 5 \mathrm{~mm}$ and cleaned with propan-2-one. The samples were single lap jointed with Acryloid B-72 while maintaining an overlapping zone of $1 \mathrm{~cm}^{2}$. The 
acrylic resin was solubilised in toluene (1:1 parts by weight) and afterwards applied with a film drawing device (wet film thickness: $100 \mu \mathrm{m})$. Test series with untreated and plasma-treated samples were prepared. The time between pre-treatment and coating did not exceed $1 \mathrm{~h}$. During drying (PELD: 8 weeks, PE-HD / PP: 6 weeks), the samples were kept under constant pressure $\left(267 \mathrm{~g} / \mathrm{cm}^{2}\right)$. After drying, the untreated and the pre-treated samples were tested back-toback at the same climatic conditions, using a tensile testing equipment (Zwick 1120, BZ 2.5 , testing speed: $50 \mathrm{~mm} / \mathrm{min}$ ). By comparing the breaking strength of untreated and pre-treated adhesives joints, changes in adhesion qualities were quantified. For each test series at least nine identical treated samples were tested. Results were evaluated statistically on the basis of calculated averages and standard derivations and are, as all results in this study, displayed with confidence intervals of $95 \%$.

\subsection{Examination of Changes in Coatability}

Changes in coatability were examined by performing pull-off tests, carried out on gouache-painted PE-HD and PS polymer plates (commercial extruded homopolymers, dimensions as described before). Gouache paint is a water-based retouching medium that is expected to exhibit good long-term water removability on plastic surfaces. For sample preparation the plates were coated with the paint (cadmium yellow, PY 35, Schmincke, wet film thickness: $50 \mu \mathrm{m})$, again by using the film drawing device. After drying (PE-HD: 4 weeks, PS: 1 week), a metal stub, $5 \mathrm{~mm}$ in diameter, was adhered to the coating using a high-viscous cyanoacrylate glue. The force required to pull the coating off was measured with the tensile tester $24 \mathrm{~h}$ after stub application. For each test series, at least eight samples with identical pre-treatment were tested. To prevent the glue itself from falsifying the results of the pull-off tests, the usage of an adhesive with low penetration capability was necessary. The acceptability of different adhesives was examined in preliminary tests by applying them on free gouache films (wet film thickness $50 \mu \mathrm{m}$ ) and analysing the chemical composition of the films backside with FTIR-ATR. The infrared spectra of the samples coated with the used high-viscous cyanoacrylate glue showed no accordant IR-absorbencies on their backside.

\subsection{Surface Temperature Measurements}

Contactless surface temperature measurements were carried out using an infrared pyrometer with double laser aiming (CT laser, Optris). During measurements, the plasma jet aimed rectangularly to a $4 \mu \mathrm{m}$ thin PP foil (XRF sample support, InstruMed inc., Atlanta) that was fixed onto an aperture plate. On its backside, the PP foil was fitted with a film of a defined emission ratio $(0.95)$. The pyrometer was positioned opposite the plasma source so that its laser aimed through the aperture opening onto the PP film's backside. The laser marked the centre of the treatment area with a spot sized as small as $1.4 \mathrm{~mm}$. Temperature values during pre-treatment were recorded during $120 \mathrm{~s}$ with a resolution of $500 \mathrm{~ms}$. This long recorded treatment time reflects a worst case scenario. The measurements were performed at a fixed operating distance of $3 \mathrm{~mm}$, while during the test runs the voltage input and the gas flow rate were varied.

\section{Results}

After pre-treatment on PP an increase in water wettability was observed as shown in Fig. 3. On the untreated, hydrophobic surface an advancing water contact angle of $100 \pm 2$ degrees was measured. Plasma treatment changed contact angle values to $46 \pm 2$ degrees.

The observed alteration in wettability is interpreted to be caused by changes in the chemical composition of the PP surface. ATR-IR spectra of plasma-treated sample surfaces revealed a considerable increase of polar, oxygen-containing functional groups. Fig. 4 shows a comparison of infrared spectra taken from untreated and pre-treated PP. The most notable changes were found in the IR-absorbance range at about $1850-1550 \mathrm{~cm}^{-1}$, known to be the absorbance region of carbonyl stretch vibrations. The intensity of two bands, situated at about $1735 \mathrm{~cm}^{-1}$ and $1631 \mathrm{~cm}^{-1}$, was significantly augmented. Bands at about $1735 \mathrm{~cm}^{-1}$ were assigned to aliphatic ketones or aldehydes. The absorbance at about $1631 \mathrm{~cm}^{-1}$ can be interpreted as carbonyl stretch vibrations of unsaturated ketones $\left(1700-1615 \mathrm{~cm}^{-1}\right)$.

Regarding the results of the tensile shear tests, the adhesion qualities of untreated PE-LD, PE-HD and PP were noticeably improved after plasma treatment (Fig. 5). The breaking force necessary to separate the PE-LD adhesive bonds showed a marked increase from $26 \pm 19$ $\mathrm{N} / \mathrm{cm}^{2}$ to $160 \pm 38 \mathrm{~N} / \mathrm{cm}^{2}$. In the case of PE-HD, a significant augmentation from $31 \pm 11 \mathrm{~N} / \mathrm{cm}^{2}$ to $215 \pm 44 \mathrm{~N} / \mathrm{cm}^{2}$ took place. In the case of PP, although the effect of the pre-treatment was less distinct, a significant improvement of the breaking force from $30 \pm 23 \mathrm{~N} / \mathrm{cm}^{2}$ to $112 \pm 32 \mathrm{~N} /$ $\mathrm{cm}^{2}$ was obtained. As found for PE-LD, the composition of the process gas influ-

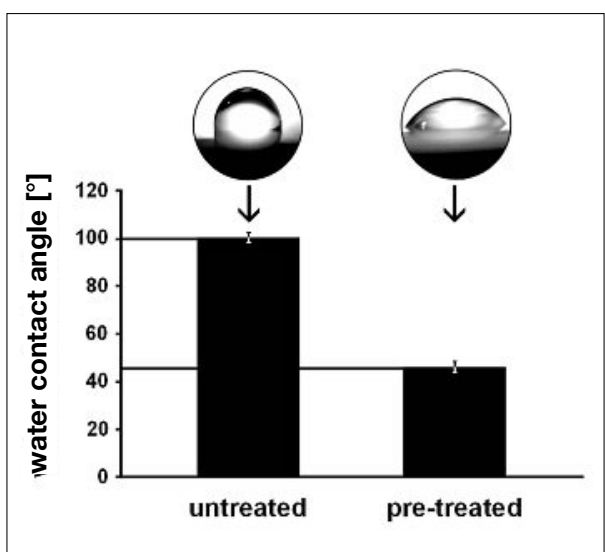

Fig. 3. Effect of the pre-treatment on the surface wettability: Advancing water contact angles on untreated and pre-treated PP samples. For each test series values were averaged over 14 similar measurements. Values are displayed here, as all results in this study, with confidence intervals of $95 \%$.

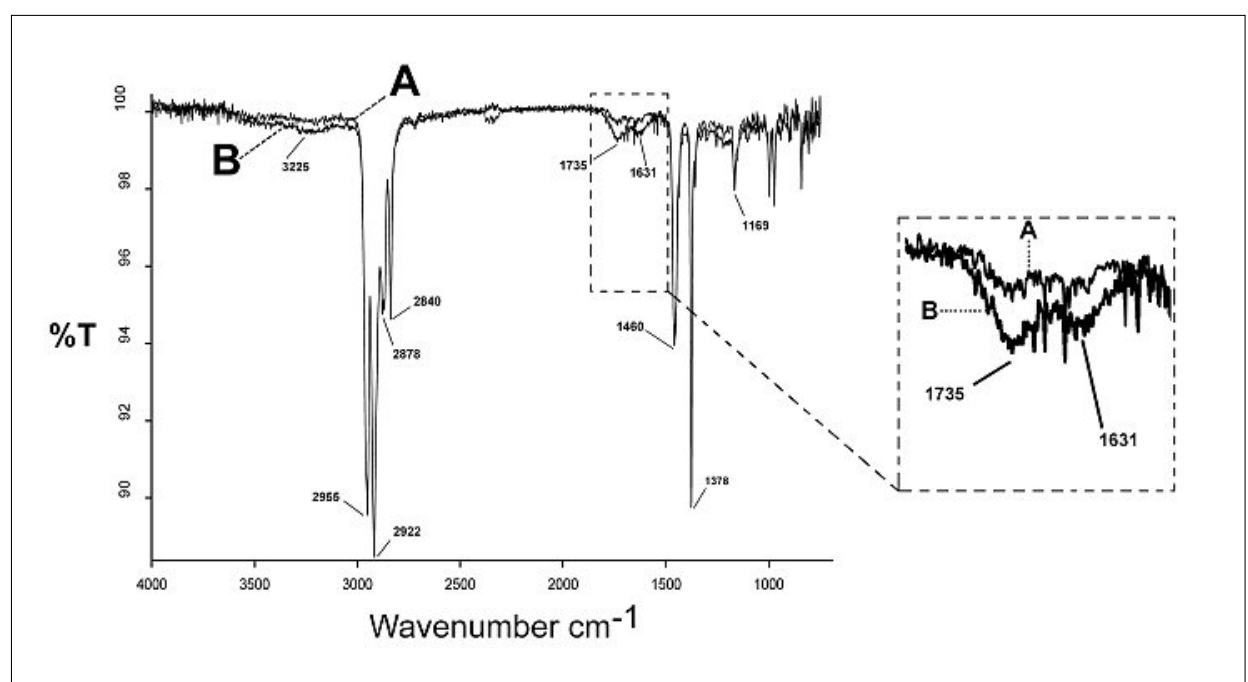

Fig. 4. Effect of the pre-treatment on the surface polarity: FTIR-ATR spectra taken from an untreated (A) and a pre-treated (B) PP surface. Most notable changes, found in the IR-absorbance range of carbonyl stretch vibrations, are displayed under magnification. 


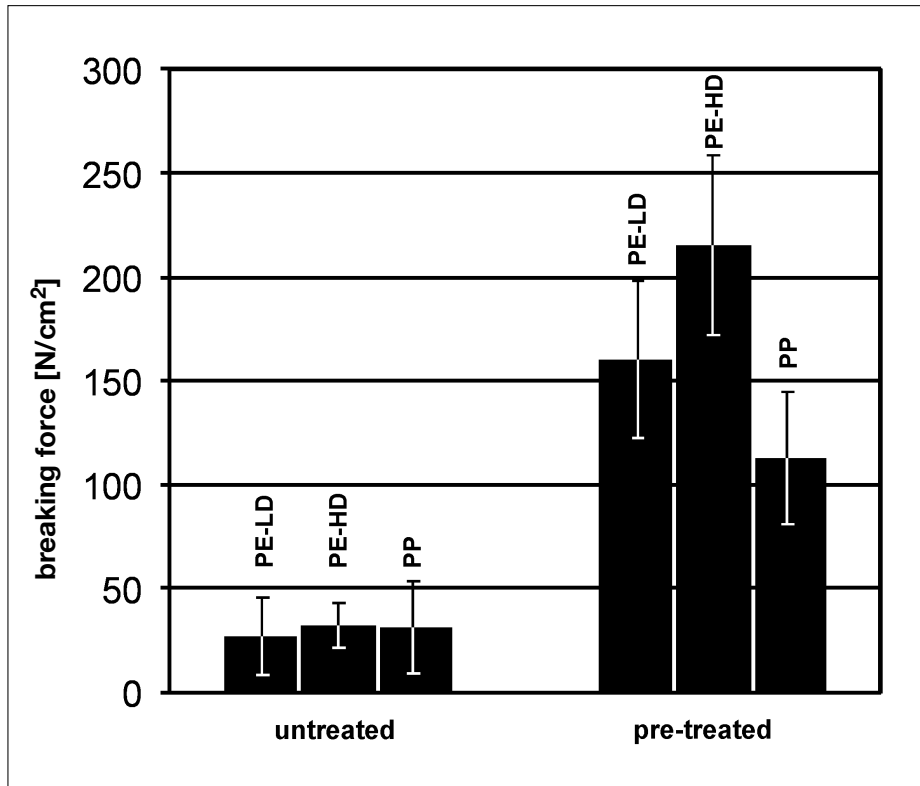

Fig. 5. Effect of the pre-treatment on the bondability of PE-LD, PE-HD and PP

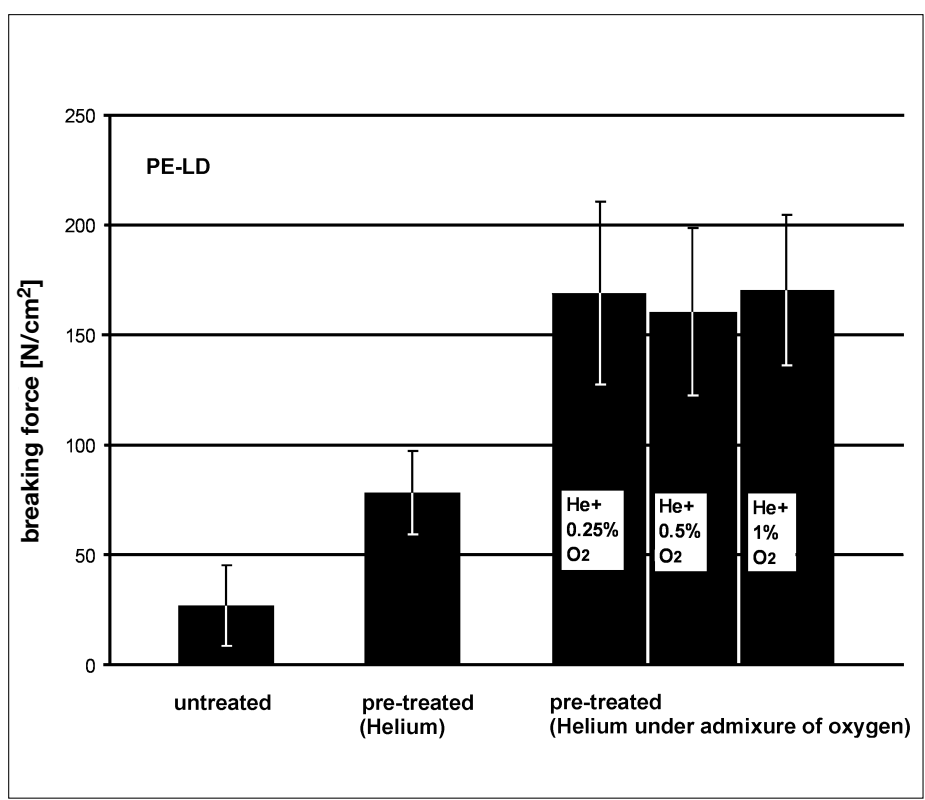

Fig. 6. Effect of the process gas on the bond strength of PE-LD adhesive joints enced the extent of adhesion improvement. The admixture of a small amount of oxygen $(0.25-1 \%)$ had a beneficial impact on increasing breaking force (Fig. 6). As expected, all untreated joints showed adhesive failure on testing. In the majority of cases the pre-treated samples showed a combination of adhesive and cohesive broken zones.

Besides this significant improvement of bondability, the applied gouache coatings also gained a considerable mechanical resilience after pre-treatment (Fig. 7). Without pre-treatment, the force required to pull the coatings off was $10 \pm 7 \mathrm{~N}$ (PEHD) and $1 \pm 3 \mathrm{~N}$ (PS), indicating that unexceptional adhesive failures took place. This very low adhesion was clearly augmented after pre-treatment to $114 \pm 34 \mathrm{~N}$ (PE-HD) and $78 \pm 30 \mathrm{~N}(\mathrm{PS})$. The pre-treated samples mostly showed cohesive failure inside the gouache coating layer, this observation confirms that adhesion qualities were improved.

As can be seen in Fig. 8, the plasma treatment increased the temperature of the pre-treated surface. To decrease this thermal load is of great importance to avoid damage during the pre-treatment of heat-sensitive substrates. In particular in the case of thin thermoplastic polymer films distortions and deformations have to be excluded. It was possible to reduce the temperature load of the pre-treated substrate by tripling the helium flow rate (Fig. 8B), whilst maintaining an oxygen admixture of $0.5 \%$. A further decrease of temperature was reached by additionally reducing the voltage input (Fig. 8C). First test results, which will be presented in a subsequent paper, indicate that also under these gentle pre-treatment conditions

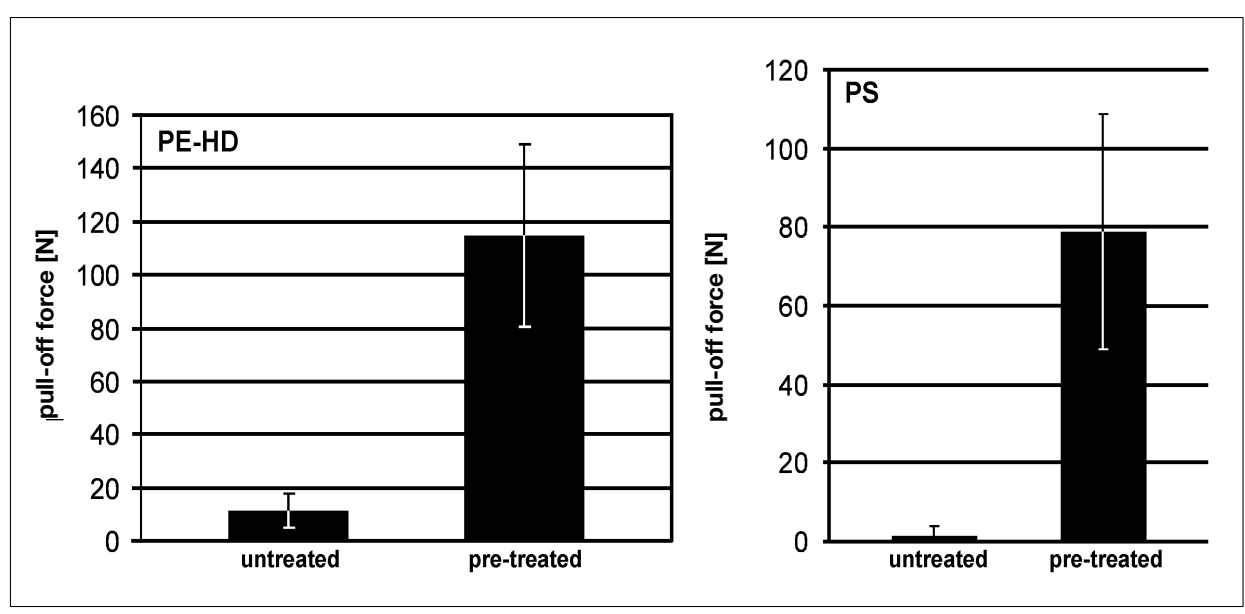

Fig. 7. Effect of the pre-treatment on the coatability of PE-HD and PS. Bar plot on the left: measured pull-off forces on PE-HD; bar plot on the right: measured pull-off forces on PS. Pre-treatment time: $0.5 \mathrm{~s} / \mathrm{mm}$.

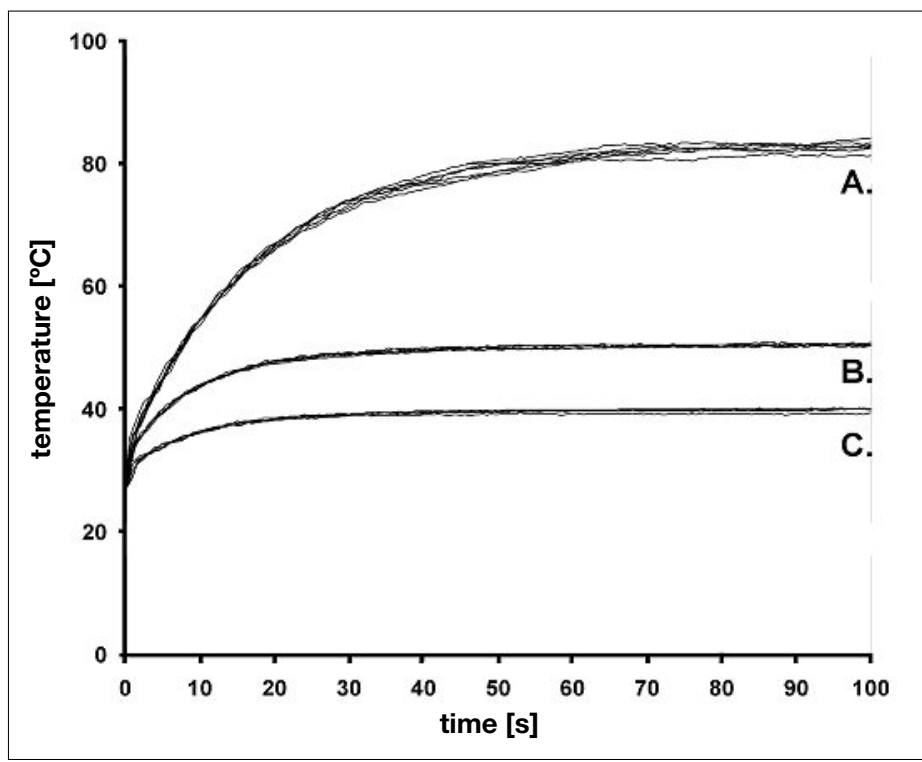

Fig. 8. Effect of different pre-treatment modes on substrate temperature. The following processing parameters were used: A) $\mathrm{He}: 1000 \mathrm{ml} / \mathrm{min}$ $\mathrm{O}_{2}: 5 \mathrm{ml} / \mathrm{min}, \mathrm{U}=1600$ volts; B) He: 3000 ml/ $\mathrm{min}, \mathrm{O}_{2}: 15 \mathrm{ml} / \mathrm{min}$; C) He: $3000 \mathrm{ml} / \mathrm{min}$ $\mathrm{U}=1600$ volts, $\mathrm{O}_{2}$ : $15 \mathrm{ml} / \mathrm{min}$, reduced voltage input level: 1000 volts. For each processing parameter seven similar measurements were performed and are displayed here. 
a significant adhesion improvement can still be achieved.

\section{Conclusions}

The developed miniaturized plasma source is a promising tool to overcome adhesion problems during conservation and restoration treatments on non-polar plastics in modern and contemporary art. The source is operable at atmospheric pressure, the plasma is applicable in a millimetre range and the pre-treatment results in a significant improvement of adhesion qualities of the tested polymers. Adhesive joint strength of pre-treated PE-LD, PE-HD and PP, bonded with the acrylic resin Paraloid B-72, were significantly improved. It is expected that also the bonding strength to other polar adhesives can be elevated, given that their dried adhesive films have an adequate cohesive strength. The tested gouache coatings also gained a considerable mechanical resilience and therefore the application of water-removable retouchings on hydrophobic plastic surfaces becomes possible. On water-insensitive plastic surfaces like PE, PP and PS, this offers a new option to obtain reversibility, an important ethical demand during conservation-restoration treatments. Particularly the use of other solvents than water can be avoided, thereby preventing solvent-induced damages (e.g. environmental stress cracking) on solventsusceptible plastics like poly(styrene). Fur- thermore this plasma source seems to be suitable for application on heat-sensitive surfaces; the thermal load caused during the pre-treatment could be minimized by reducing the voltage input and augmenting the process gas flow rate. Finally it must be noted that an important chemical mechanism during this pre-treatment seems to be surface oxidation. This conclusion is made with respect to the formation of polar, oxygen-containing functional groups, detected by infrared spectrometric analysis (FTIRATR). Before implementation of the device in conservation and restoration practice, undesirable side effects on the long-term aging properties of the pre-treated polymers, especially concerning their oxidative stability, have to be excluded. First studies on changes in oxidation stability are in progress, whereas the pre-treated polymers are analysed by chemiluminescence analysis and electro-paramagnetic resonance spectroscopy (EPR). The aim of these further studies is to assure a minimal intervention into the artwork's material integrity.

\section{Acknowledgements}

The author would like to thank the workgroup of Dr. C. Hollenstein (Plasma Physics Research Centre CRPP, Swiss Federal Institute of Technology, Lausanne) for infrastructure support and the very valuable and inspiring technical discussions. Special thanks go beside Dr. C. Hollenstein to Dr. Jean-Luc Dorier, Dr. Alban Sublet and Samantha Pavon. The author would also like to thank the workgroup of em. Prof. Dr. Döbele (Institute for Laser- and
Plasma Physics, University of Duisburg-Essen), especially Dr. Stephan Reuter. The author also thanks Marc Egger, Fabian Käser and Dr. Stefan Wülfert (University of Applied Science Berne, University of the Arts) and Dr. Walter Caseri (ETH Zürich) for their worthwhile collaboration. This project could not have been completed without the financial support of the University of Applied Science Berne.

Received: October 8, 2008

[1] D.M. Brewis, I. Mathieson, 'Adhesion and Bonding to Polyolefins', Rapra Review Report 143, Rapra Technology Ltd., Shawbury, 2002.

[2] J. H. Lee, H. G. Kim, G. S. Khang, H. B. Lee, M. S. Jhon, J. Colloid Interface Sci. 1992, 151, 563.

[3] A. R. Blythe, D. Briggs, C. R. Kendall, D. G. Rance, V. J .I. Zichy, Polymer 1978, 19 , 1273.

[4] L. J. Gerenser, J. F. Elman, M. G. Mason, J. M. Pochan, Polymer 1985, 26, 1162.

[5] M. Strandal, D. A. I. Goring, Polymer Engineering and Science 1977, 17, 38.

[6] M. Morra, E. Occhiello, L. Gila, F. Garbassi, J. Adhesion 1990, 33, 77.

[7] P. Gatenholm, C. Bonnerup, E. Wallström, J. Adhesion Sci. Technol. 1990, 4, 817.

[8] S. Wu, 'Polymer Interface and Adhesion', Marcel Dekker, New York, Basel, 1982. 